\title{
Development of emotional sweating in the newborn infant
}

\author{
V A HARPIN AND N RUTTER \\ Department of Neonatal Medicine and Surgery, Nottingham City Hospital
}

SUMMARY Sweating from the palm and sole occurs independently of ambient temperature but is influenced by emotional factors. It thus provides a useful objective measure of emotional state. The development of this emotional sweating in the newborn was investigated by measuring palmar water loss and relating it to the infant's state of arousal. Altogether 433 individual measurements were made on 124 babies of gestational age 25 to 41 weeks and postnatal age 15 hours to 9 weeks. Palmar water loss was also recorded continuously in 22 infants undergoing heel prick for routine blood sampling. In babies of 37 weeks' gestation or more, there was a clear relationship between palmar water loss and arousal from the day of birth, and by the third week levels on vigorous crying were comparable with those of an anxious adult. Less mature babies did not show emotional sweating at birth; it was first seen at the equivalent of 36 to 37 weeks' gestation regardless of maturity. Continuous recordings confirmed the cross-sectional data and illustrated the abrupt nature of the response. Emotional sweating could be a useful tool for the assessment of emotional state of the newborn.

Sweating from most areas of the body occurs in response to a warm environment and is an important defence against overheating. By contrast, sweating from the palm of the hand and the sole of the foot is largely unaffected by changes in ambient temperature. It is determined instead by emotional factors, being increased by pain, fear, anxiety, and concentration and decreased by contentment, relaxation, and sleep. This is termed emotional or mental sweating. It is widely used as a tool in psychological medicine and research, providing an objective measurement of emotional state. The newborn infant may experience pain or pleasure in response to different stimuli but his limited range of behaviour makes it difficult to assess such emotions. An objective measure of emotional well-being would clearly be useful as a tool in neonatal research. Previous work however suggests that emotional sweating does not occur in the newborn until the second month of life. ${ }^{12}$

In a recent study of the development of thermal sweating in the newborn, we noted that water loss from the palm and the sole was high in mature infants from birth. Although unaffected by changes in ambient temperature, it did vary with the infant's state of arousal. ${ }^{3}$ This suggests that emotional sweating does occur in the newborn. We therefore undertook this study to examine in detail the development of emotional sweating in the newborn, with particular reference to gestation and postnatal age. Since our original study showed that water loss from the palm was greater and varied more with the state of arousal than water loss from the sole, the former was chosen as the indicator of emotional sweating.

\section{Method}

Palmar water loss was measured using an Evaporimeter (Evaporimeter Ep1, Servomed, Sweden). This device measures the gradient of water vapour pressure close to the skin surface, which is directly proportional to the rate of evaporation of water from the skin. Its use has previously been described in detail. ${ }^{4-8}$ Each infant was studied in an unhumidified incubator or in a cot. The hand was first unclenched, taking care not to touch the palm, and exposed to the surrounding air so that any trapped moisture could evaporate. The probe of the instrument was then gently rested on the centre of the infant's palm until a steady reading of water loss in $\mathrm{g} / \mathrm{m}^{2}$ per hour was obtained. Care was taken to avoid draughts and to prevent the baby from breathing over the probe since these result in unstable readings. The state of arousal of the infant at the time of the measurement was assessed using a simple scoring 
system, ranging from deep non-rapid eye movement (non-REM) sleep to vigorous crying (Table 1). Altogether 433 individual measurements were made on 124 infants whose gestational age ranged from 25 to 41 weeks, who were between 15 hours and 9 weeks old (Table 2). Gestational assessment was calculated from the first day of the mother's last menstrual period and estimated by means of physical assessment. ${ }^{9}$ If these differed by more than 2 weeks the infant was not included in the study.

In an additional 22 infants palmar water loss was recorded continuously by attaching a chart recorder to the Evaporimeter. Recording began with the infant asleep or quiet; a heel prick for routine blood sampling was then performed. The change in palmar water loss was measured as the infant awoke, cried, and settled again. These infants ranged in gestation from 25 to 40 weeks and were aged between 4 and 68 days.

The environmental temperature varied between 26 and $37^{\circ} \mathrm{C}$ and the relative humidity between 30 and $54 \%$.

\section{Results}

Infants of at least 37 weeks' gestation showed palmar water losses which varied greatly with state of arousal, being lowest in non-REM sleep and highest during crying (Fig. 1 and Table 3). All mature infants demonstrated this, the earliest study being at age 15 hours. The increase in palmar water loss with arousal became more pronounced as the infants grew older.

Table 1 Scoring system used to assess state of arousal

\begin{tabular}{rll}
\hline-1 & $=$ & deep, non-REM sleep \\
0 & $=$ REM sleep \\
1 & $=$ & awake/asleep, minor movement with occasional general \\
2 & $=$ & movement \\
3 & $=$ & minor crying, continuous movements \\
\end{tabular}

Table 2124 babies in whom emotional sweating was studied

\begin{tabular}{lllll}
\hline $\begin{array}{l}\text { Gestation } \\
\text { (weeks) }\end{array}$ & $\begin{array}{l}\text { Number } \\
\text { of } \\
\text { babies }\end{array}$ & $\begin{array}{l}\text { Number } \\
\text { of } \\
\text { measure- } \\
\text { ments }\end{array}$ & $\begin{array}{l}\text { Postnatal } \\
\text { age range }\end{array}$ & Birthweight $(k g)$ \\
\cline { 4 - 4 } & & 102 & 15 hrs-63 days & $3 \cdot 05(1 \cdot 58-4 \cdot 84)$ \\
37 or more & 41 & 94 & $1-26$ days & $2 \cdot 18(1 \cdot 01-3 \cdot 2)$ \\
$34-36$ & 24 & 132 & $1-68$ days & $1 \cdot 63(1 \cdot 01-2 \cdot 2)$ \\
$30-33$ & 33 & 132 Range \\
$25-29$ & 26 & 105 & $1-65$ days & $1 \cdot 09(0 \cdot 72-1 \cdot 49)$ \\
\hline
\end{tabular}

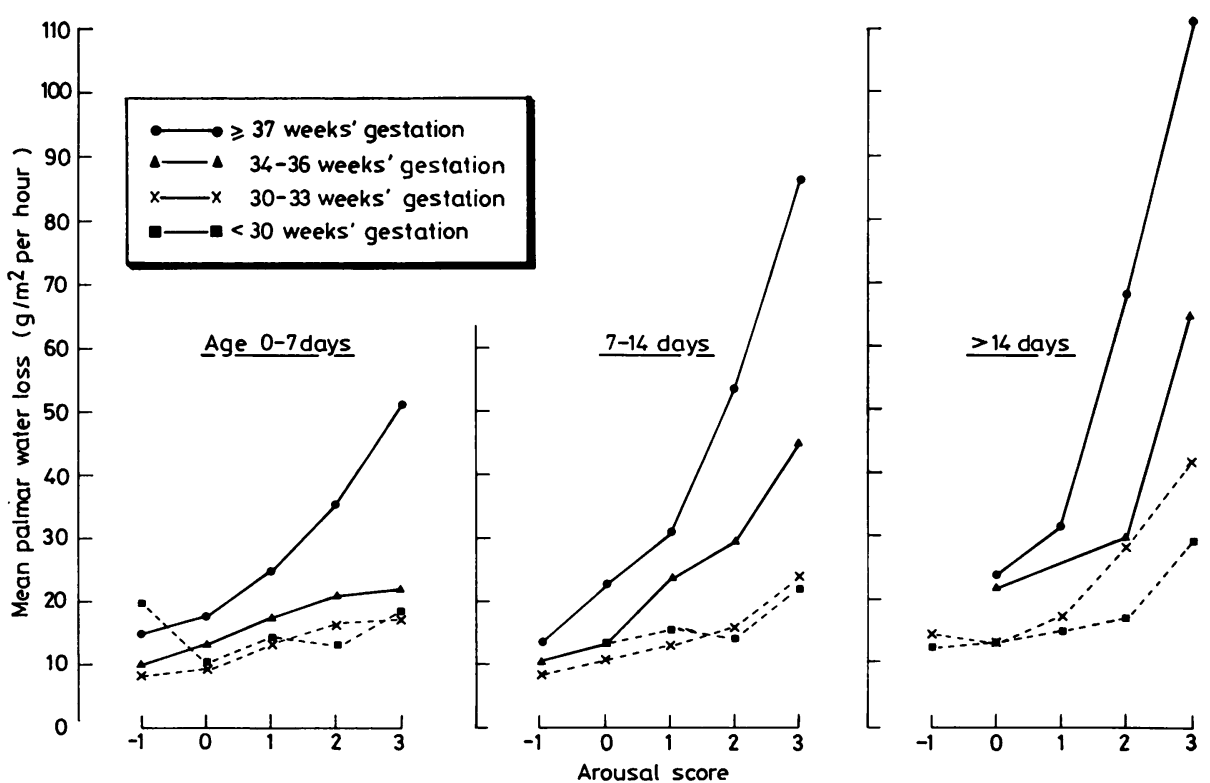

Fig. 1 Mean palmar water loss of infants in different states of arousal, according to gestation and postnatal age. Increasing palmar water loss with arousal is affected by both gestation and postnatal age. It first appears at the equivalent of 36 to 37 weeks' gestation (gestation + postnatal age) and is absent before this stage. 
Table 3 Palmar water loss (mean and range) of infants in 4 gestational age groups when in rapid eye movement sleep and when crying vigorously. The maturation effect of increasing postnatal age is seen

\begin{tabular}{|c|c|c|c|c|c|}
\hline \multirow{3}{*}{$\begin{array}{l}\text { Gestation } \\
\text { (weeks) }\end{array}$} & \multirow[t]{3}{*}{ Postnatal age } & \multicolumn{4}{|c|}{ Palmar water loss ( $\mathrm{g} / \mathrm{m}^{2}$ per hour $)$} \\
\hline & & \multicolumn{2}{|c|}{ In REM sleep } & \multicolumn{2}{|c|}{$\begin{array}{l}\text { When vigorously } \\
\text { crying }\end{array}$} \\
\hline & & Mean & Range & Mean & Range \\
\hline 37 or more & $\begin{array}{l}\text { 1st week } \\
\text { 2nd week } \\
>2 \text { weeks }\end{array}$ & \multicolumn{2}{|c|}{$\begin{array}{l}18(12-25) \\
24(20-26) \\
24(20-28)\end{array}$} & \multicolumn{2}{|c|}{$\begin{array}{l}50(25-95) \\
85(65-120)\end{array}$} \\
\hline $34-36$ & $\begin{array}{l}\text { 1st week } \\
\text { 2nd week } \\
>2 \text { weeks }\end{array}$ & $\begin{array}{l}13(6- \\
13(10 \\
22(20\end{array}$ & & \multicolumn{2}{|c|}{$\begin{array}{l}22(12-35) \\
45(32-66) \\
65(30-90)\end{array}$} \\
\hline 30-33 & $\begin{array}{l}\text { 1st week } \\
\text { 2nd week } \\
>2 \text { weeks }\end{array}$ & \multicolumn{2}{|c|}{$\begin{array}{l}11(9-14) \\
13(8-18)\end{array}$} & \multicolumn{2}{|c|}{$\begin{array}{l}24(19-30) \\
42(25-75)\end{array}$} \\
\hline 25-29 & $\begin{array}{l}\text { 1st week } \\
\text { 2nd week } \\
>2 \text { weeks }\end{array}$ & $\begin{array}{l}10(8- \\
12(6- \\
13(7-\end{array}$ & & \multicolumn{2}{|c|}{$\begin{array}{l}20(19-25) \\
30(20-32)\end{array}$} \\
\hline
\end{tabular}

Infants of 34 to 36 weeks' gestation showed only slight increases in palmar water loss with increasing arousal in the first week of life. However, by the second week the variation was pronounced in some infants and in later studies results were similar to term infants in the first week of life.

Infants of 30 to 33 weeks' gestation showed minimal variation in palmar water loss in the first and second weeks of life. Thereafter, a high palmar water loss was demonstrated in aroused infants when they reached the equivalent of 36 to 37 weeks' gestation.

Infants of less than 30 weeks' gestation similarly showed little variation in palmar water loss in the immediate newborn period. Again the variation with arousal began to appear when the infant reached an equivalent gestational age of 36 to 37 weeks.

The continuous recordings of palmar water loss confirmed the cross-sectional data. In term infants a striking increase was seen when the infant cried, decreasing when the infant settled (Fig. 2). The change in palmar water loss in mature infants is abrupt, occurring within seconds of increased arousal and falling equally rapidly as the baby settles. Again no such response was seen in infants who had not reached the equivalent of 36 to 37 weeks' gestation. Fig. 3 shows serial recordings of an

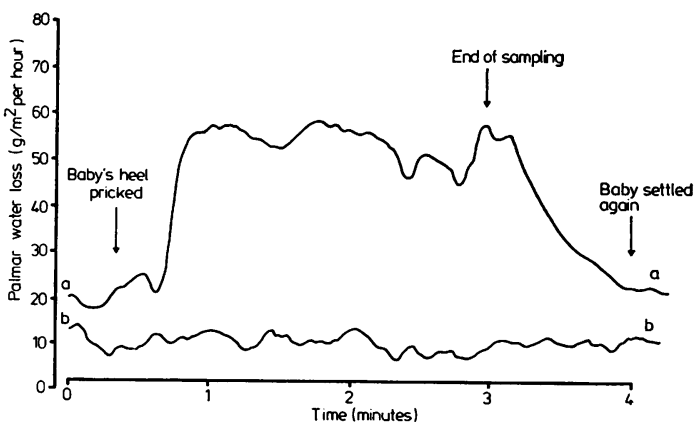

Fig. 2 Continuous recording of palmar water loss during heel prick for routine blood sampling (a) in a term baby aged 6 days, and (b) in a baby of 35 weeks' gestation aged 4 days. Initially both infants were asleep or quiet; after heel prick they cried vigorously and then settled again. The term baby shows an abrupt increase in palmar water loss as a result of the painful stimulus which falls rapidly as the infant settles. The less mature infant did not demonstrate this response.

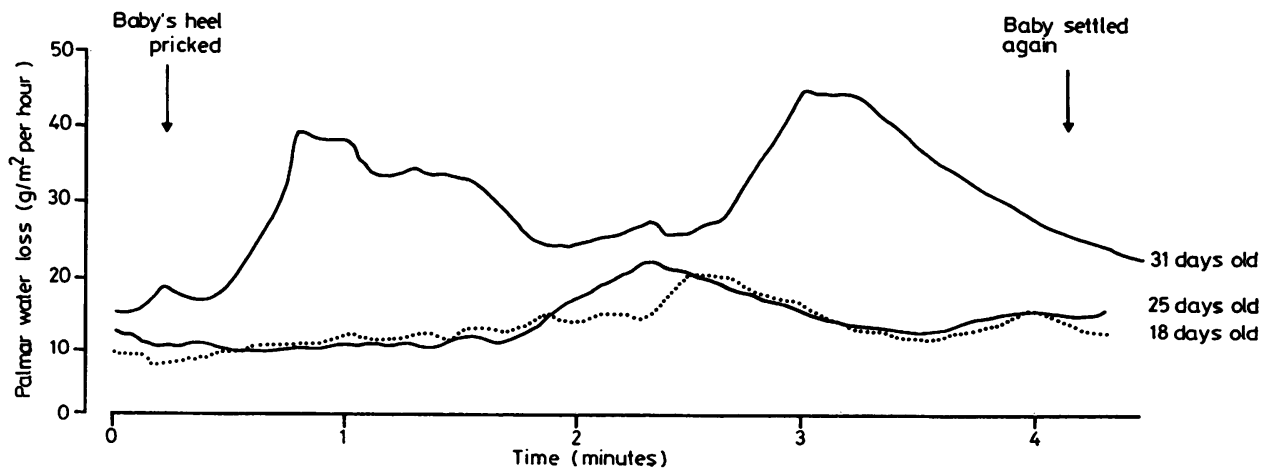

Fig. 3 Serial recordings of palmar water loss during heel-prick blood sampling on an infant of 32 weeks' gestation. Recordings at 18 and 25 days of age show only a minimal and slow increase in palmar water loss with arousal. At age 31 days (the equivalent of $36+$ weeks' gestation) a striking, abrupt, increase in palmar water loss is seen when the infant cries. 
infant of 32 weeks' gestation who first demonstrates an increase in palmar water loss with arousal at age 31 days.

\section{Discussion}

It seems likely that palmar water loss in the newborn, as in adults, is due to sweating rather than to the passive diffusion of water through the epidermis. Rates of water loss in sleeping term infants are considerably higher from the palms than from other sites in the absence of thermal sweating, 58 yet the epidermis of palmar skin is thicker than elsewhere. We presume that background sweating from the palm is occurring perpetually, regardless of state of arousal. Such background sweating has been detected in the mature newborn by the staining of active sweat pores. $^{2}$ We have shown that palmar water loss varies with arousal state in mature infants and that this response is both abrupt and pronounced. Emotional sweating therefore does occur in the newborn.

The purpose of sweating from paws or palms in response to stress is not fully understood. Adelman et al. ${ }^{10}$ demonstrated elegantly that sweating from the paws of 4 species of mammal increased on exercise, resulting in increased friction between paw and ground as the animal ran. They suggested that this plays an important role in the animal's flight from danger. Kuno ${ }^{11}$ and his colleagues investigated emotional sweating in man, differentiating it from thermal sweating by showing that palmar sweat glands responded little to rise in ambient temperature but rapidly to changes in emotion. They demonstrated that emotional sweating has no latent period before onset and that it subsides rapidly once thestimulation ends. Emotional sweating in infants was first studied by Uchino $^{1}$ using a ventilated capsule. He was unable to demonstrate its presence in term infants until at least 33 days of age, but the method is insensitive. Verbov and Baxter, ${ }^{2}$ using an o-phthaldialdehyde solution, demonstrated sweating on the palm in term infants in the first few days of life but they did not demonstrate changes with arousal.

We have shown that emotional sweating on the palm does occur in mature newborn babies from the day of birth It reaches levels on crying which are comparable with those found in anxious adults by about the equivalent of 43 weeks' gestation. Emotional sweating first appears at the equivalent of 36 to 37 weeks' gestation, regardless of gestation at birth, so that its development is largely unaffected by postnatal existence. By contrast, the development of thermal sweating is markedly hastened by post- natal existence so that almost every baby is able to sweat in response to a high ambient temperature by age 2 weeks, regardless of gestation. ${ }^{3}$ Cholinergic fibres of the sympathetic nervous system are affected in both these sweating mechanisms, but they differ in that thermal sweating is controlled by the hypothalamus whereas the emotional sweating response involves a higher centre in the premotor cortex. ${ }^{11} 12$

Emotional sweating is the basis of the galvanic skin response: the increase in skin conductance on the palm or sole which occurs during arousal. ${ }^{13}$ Skin conductance is widely used by psychologists in the study of stress; ${ }^{14}$ the 'lie detector' is one of the best known examples of its use. It has been used to examine the stress of adults visiting the dentist ${ }^{15}$ and of children admitted to hospital for routine surgery. ${ }^{16}$ This present study has shown that emotional sweating occurs in the newborn from an equivalent of 36 to 37 weeks' gestation and is readily measured. It is therefore possible to study stress in the newborn in an objective, quantifiable way. One area in which stress could be thus studied is in the field of neonatal intensive care. Although the absence of emotional sweating in preterm infants limits its usefulness, it could be studied in more mature infants undergoing intensive care. The stress caused by painful practical procedures could be examined and the effect of measures to reduce such stress-such as modification of a technique or the administration of analgesia-could be studied objectively. Clearly a struggling, screaming infant is in pain and measurement of palmar water loss is not necessary to recognise this. Infants who receive intensive care however are not always able to make such clear demonstrations of discomfort. Endotracheal tubes, for example, remove the ability to cry and sedatives and paralysing agents may reduce movement without reducing pain. The technique of measurement of emotional sweating may also prove useful in the study of less obvious stress or indeed in the study of emotional contentment in the newborn. As such it might be of interest to the child psychologist studying infant development or the interaction between the infant and his mother.

V A $\mathrm{H}$ was supported by a grant from the Medical Research Council of Great Britain.

\section{References}

1 Uchino S. Cited by $\mathrm{Y}$ Kuno in Human perspiration. Springfield: Thomas, 1956.

2 Verbov J, Baxter J. Onset of palmar sweating in newborn infants. Br J Dermatol 1974; 90: 269-76. 
${ }^{3}$ Harpin V A, Rutter N. Sweating in preterm babies. J Pediatr 1982; 100: 614-9.

4 Nilsson G E. Measurement of water exchange through the skin. Med Biol Eng Comput 1977; 15: 209-18.

5 Hammarlund K, Nilsson G E, Oberg P A, Sedin G. Transepidermal water loss in newborn infants. I. Relation to ambient humidity and site of measurement and estimation of total transepidermal water loss. Acta Paediatr Scand 1977; 66: 553-62.

6 Hammarlund K, Nilsson G E, Oberg P A, Sedin G. Transepidermal water loss in newborn infants. II. Relation to activity and body temperature. Acta Paediatr Scand 1979; 68: 371-6.

7 Hammarlund K, Sedin G. Transepidermal water loss in newborn infants. III. Relation to gestational age. Acta Paediatr Scand 1979; 68: 795-801.

8 Rutter N, Hull D. Water loss from the skin of term and preterm babies. Arch Dis Child 1979; 54: 858-68.

- Farr V, Kerridge D F, Mitchell R G. The value of some external characteristics in the assessment of gestational age at birth. Dev Med Child Neurol 1966; 8: 657-60.

10 Adelman S, Taylor C R, Heglund N C C. Sweating on paws and palms: what is its function? Am J Physiol 1975; 229: $1400-2$.
11 Kuno Y. Human perspiration. Springfield: Thomas, 1956.

12 Sinclair D. Motor nerves and reflexes. In: Jarrett A, ed. The physiology and pathophysiology of the skin. Vol. 2. London: Academic Press, 1973: 475-508.

13 Lader M H, Montagu J D. The psycho-galvanic reflex: a pharmacological study of the peripheral mechanism. $J$ Neurol Neurosurg Psychiatr 1962; 25: 126-33.

14 Greenfield N S, Sternbach R A, eds. Handbook of psychophysiology. New York: Holt, Rinehart, 1972.

15 Early C E, Kleinknecht R A. The palmar sweat index as a function of repression-sensitization and fear of dentistry. $J$ Consult Clin Psychol 1978; 46: 184-5.

16 Johnson P A, Stockdale D F. Effects of puppet therapy on palmar sweating of hospitalized children. Johns Hopkins Med J 1975; 137: 1-5.

Correspondence to Dr N Rutter, Department of Neonatal Medicine and Surgery, Nottingham City Hospital, Hucknall Road, Nottingham NG5 1PD.

Received 1 June 1982 